\title{
APLICACIÓN DE REDES NEURONALES PARA EVALUAR EL ESTADO FUNCIONAL DE PACIENTES CON ESCLEROSIS MÚLTIPLE
}

\author{
Janire Otamendi, Asier Zubizarreta, Itziar Cabanes, Eva Portillo \\ Depto. de Ingeniería de Sistemas y Automática, Universidad del País Vasco (UPV/EHU) \\ janire.otamendi@ehu.eus
}

\begin{abstract}
Resumen
La Esclerosis Múltiple es una enfermedad neurodegenerativa que actualmente no tiene cura. Sin embargo, las terapias personalizadas han demostrado ralentizar o incluso detener la evolución de la enfermedad, mejorando asi la calidad de vida de los pacientes. Para diseñar dichas terapias, es esencial conocer el estado funcional de cada paciente, lo cual suele ser una tarea laboriosa. Ante esta situación, en este trabajo se propone el desarrollo de un clasificador de estado de pacientes de Esclerosis Múltiple. Para ello, se hace uso de redes neuronales artificiales, las cuales utilizan una base de datos obtenida mediante una contera inteligente para desarrollar un sistema que permita determinar el estado funcional de los pacientes.
\end{abstract}

Palabras clave: Esclerosis Múltiple, Contera sensorizada, Valoración pacientes, Clasificador de estado funcional, Machine Learning, Redes neuronales artificiales.

\section{INTRODUCCIÓN}

La Esclerosis Múltiple (EM) es una enfermedad neurodegenerativa, progresiva y crónica que afecta a unos 2,5 millones de personas en el mundo [10]. A pesar de tener una incidencia notablemente inferior a otras afecciones como el ictus o el Alzheimer, la EM tiene un impacto socioeconómico muy superior, debido a que afecta a personas desde una edad muy temprana (media 30 años) y evoluciona durante muchos años.

Aunque los síntomas de la enfermedad varían en función del área afectada, la fatiga y la disfunción motora son las más comunes, por lo que más de un $80 \%$ de los pacientes requiere de un dispositivo de ayuda técnica (muleta, bastón, andador) tras 15 años desde el primer brote de la enfermedad [11].

En la actualidad, no existe cura para la EM, sin embargo, estudios recientes han demostrado que una adecuada terapia de rehabilitación puede ralentizar o incluso detener la evolución de la enfermedad, mejorando la calidad de vida de los pacientes [6]. Para dicho fin, es fundamental la per- sonalización de la terapia al estado particular de cada paciente y esto hace que, realizar una adecuada valoración funcional de dichos pacientes se torne esencial.

Tradicionalmente, la valoración de los pacientes de EM se ha realizado utilizando diferentes escalas clínicas normalizadas. La Short Physical Performance Battery (SPPB) es una de ellas, la cual consiste en la realización de varias pruebas que tratan de evaluar el equilibrio, la fuerza y la velocidad de la marcha del paciente. La puntuación en esta escala oscila entre 0 y 12 , siendo 12 la mejor puntuación. Sin embargo, realizar las pruebas para poder obtener estos valores es una tarea laboriosa, con lo que la periodicidad entre las mismas es elevada [5].

Una de las posibles soluciones a este problema consiste en monitorizar a los pacientes y extraer indicadores que puedan ayudar a evaluar su estado de una manera objetiva. De esta forma, el terapeuta dispone de información sin necesidad de realizar todas las pruebas, para así rediseñar la terapia en caso de detectar variaciones en el estado funcional del paciente.

Existen diversos dispositivos tecnológicos para la monitorización. La tendencia actual es utilizar dispositivos vestibles [12], los cuales se basan en sensores inerciales (acelerómetros, giróscopos) [7]. Aunque estos dispositivos son ampliamente utilizados en diversas aplicaciones, presentan inconvenientes cuando se utilizan en pacientes, dado que los métodos que se utilizan para mantenerlos fijados al cuerpo pueden resultar invasivos para éstos, sobre todo cuando presentan un deterioro en su capacidad motora.

Debido a ello, en los últimos años, se han propuesto alternativas vestibles de menor invasividad, como el uso de pulseras, relojes o móviles inteligentes [4]. Sin embargo, en el caso de pacientes de EM, los movimientos parásitos de los mismos han lastrado su aplicabilidad.

Con el objetivo de solucionar los problemas mencionados, recientes estudios han propuesto incluir sensores en dispositivos de uso cotidiano, como muletas, bastones o andadores, lo cual ha de- 
mostrado ser una alternativa eficaz para la captura de datos $[3,9]$. En este sentido, destaca el desarrollo de la Contera Sensorizada realizada por el Grupo de Sensorización Virtual para Bioingeniería [2], que constituye el punto de partida de este trabajo.

Una vez capturados los datos, es necesario procesarlos y extraer indicadores para proporcionar la información adecuada al terapeuta. En el ámbito de la EM ésta es un área apenas explorada. Los estudios realizados $[12,13]$ analizan principalmente las correlaciones entre las variables capturadas y el estado clínico del paciente medida con escalas clínicas, lo cual es útil para realizar la selección de variables significativas, pero aporta información poco procesada y en ocasiones, contradictoria, a los terapeutas.

En esta fase, el uso de las técnicas de Machine Learning (ML) se torna fundamental. En otras áreas de la salud, el procesamiento inteligente de datos ha permitido grandes avances, dado su potencial para trabajar con datos complejos y de diversa naturaleza, y su capacidad para generar modelos de comportamiento e incluso para poder extrapolar tendencias $[8,14]$.

En este trabajo se propone un clasificador del estado del paciente basado en redes neuronales, que en base de una serie de características obtenidas de los datos capturados por una contera inteligente, estima el estado del paciente según la escala SPPB. La aproximación propuesta es evaluada considerando los datos de 59 pruebas realizadas con pacientes de Esclerosis Múltiple en diferentes estadios de su enfermedad.

El resto del artículo se estructura de la siguiente manera: en la Sección 2, se presenta el prototipo de Contera Sensorizada usada para la captura de datos; la Sección 3 detalla la base de datos usada para entrenar la red neuronal; la Sección 4 define el diseño del clasificador; mientras que la Sección 5 muestra los resultados del sistema diseñado. Finalmente, la Sección 6 detalla las ideas más relevantes.

\section{CONTERA SENSORIZADA}

Conociendo las ventajas que ofrece utilizar un dispositivo de ayuda técnica para la monitorización y captura de datos en pacientes con EM, en este trabajo, se ha empleado la contera sensorizada presentada en [2] (Figura 1) para obtener la información que compone la base de datos. Cabe mencionar que, a diferencia de muletas o bastones sensorizados que se plantean en algunos de los trabajos citados [3, 9], esta contera puede ser acoplada al dispositivo de ayuda técnica que utilice el paciente, lo cual hace que éste no tenga que adaptarse a uno nuevo.

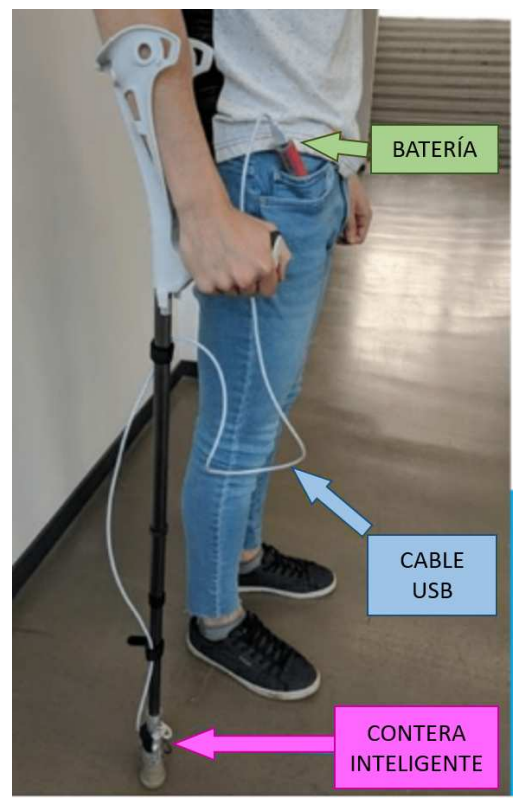

Figura 1: Contera sensorizada

La contera sensorizada se compone de un dispositivo de adquisición de datos y tres sensores (un sensor de fuerza, un barómetro y una Unidad de Medición Inercial o IMU) integrados en una estructura de aluminio.

El sensor de fuerza proporciona información sobre la fuerza axial que ejerce el paciente, el barómetro indica la presión atmosférica, permitiendo estimar la altura relativa del dispositivo, y finalmente, la Unidad de Medición Inercial ofrece los valores de la aceleración lineal, de la velocidad angular y del campo magnético en los ejes $x, y, z$ locales de la muleta. Este último elemento, además de proporcionar las señales mencionadas, también integra un algoritmo que permite estimar los ángulos de Euler en un sistema de referencia global, los cuales se emplean para calcular los ángulos entre el cuerpo del paciente y la contera en los planos anteroposterior y lateromedial [2].

\section{BASE DE DATOS}

El objetivo del presente trabajo es desarrollar un clasificador capaz de valorar a pacientes de EM, con lo que es necesario disponer de una base de datos generada a partir de pruebas realizadas con personas que padecen dicha enfermedad. Así, en esta sección, se presentan por un lado las pruebas que se han llevado a cabo (sección 3.1) y por el otro, el procesamiento de datos realizado para obtener la base de datos que se empleará en las siguientes fases (sección 3.2). 


\subsection{Pruebas con pacientes de EM}

En este trabajo, las pruebas para generar la base de datos se han llevado a cabo en la Asociación de Esclerosis Múltiple de Bizkaia (ADEMBI), con la aprobación del Comité de Ética PS2018017. Dichas pruebas han sido realizadas en tres sesiones, en las cuales han participado un total de 30 pacientes. Sin embargo, algunos de estos pacientes han colaborado en más de una sesión, con lo que se ha conseguido una base de datos que incluye la información de 59 pruebas. La Tabla 1 resume las pruebas realizadas, incluyendo la distribución de pacientes según su escala SPPB, obtenida mediante la evaluación de los terapeutas.

Tabla 1: Número de pruebas, y número de ventanas para entrenamiento y test.

\begin{tabular}{|c|c|c|c|}
\hline $\begin{array}{c}\text { Valor } \\
\text { SPPB }\end{array}$ & $\begin{array}{c}\text { Número } \\
\text { de } \\
\text { pruebas }\end{array}$ & $\begin{array}{c}\text { Muestras } \\
\text { de entre- } \\
\text { namiento }\end{array}$ & $\begin{array}{c}\text { Muestras } \\
\text { de } \\
\text { test }\end{array}$ \\
\hline 0 & 0 & 0 & 0 \\
1 & 2 & 43 & 0 \\
2 & 0 & 0 & 0 \\
3 & 4 & 62 & 15 \\
4 & 4 & 55 & 26 \\
5 & 5 & 65 & 19 \\
6 & 10 & 110 & 40 \\
7 & 7 & 64 & 25 \\
8 & 7 & 51 & 25 \\
9 & 11 & 100 & 34 \\
10 & 4 & 35 & 10 \\
11 & 4 & 31 & 11 \\
12 & 1 & 10 & 0 \\
\hline Total & 59 & 626 & 205 \\
\hline
\end{tabular}

En cada sesión, los participantes han tenido que realizar dos repeticiones de una única prueba que consiste en andar 10 metros en línea recta a una velocidad cómoda según su estado, y lo más constante posible, con la contera inteligente acoplada a su dispositivo de ayuda técnica.

\subsection{Procesamiento de datos}

Una vez realizadas las pruebas, es necesario un adecuado procesamiento de datos, el cual, en este caso, está dividido en tres fases: recortar las pruebas, realizar la segmentación y caracterizar cada segmento para obtener la información que pueda ser utilizada en una red neuronal.

La primera fase consiste en eliminar las señales correspondientes al primer y último metro de cada repetición, ya que éstos corresponden respectivamente a las fases de aceleración y deceleración.

A continuación, en un segundo paso, se realiza la segmentación de señales empleando el procedi- miento que se define en [1], el cual se basa en los ciclos de uso de la muleta. Mediante esta técnica, las señales proporcionadas por los sensores de la contera son divididos en ventanas secuenciales que están asociadas a dichos ciclos. Estos ciclos están compuestos por una fase de apoyo (en el que la contera está en contacto con el suelo) y una fase de movimiento (en el que no existe contacto entre la contera y el suelo), los cuales son fácilmente detectables mediante la señal proporcionada por el sensor de fuerza (Figura 2). Por lo tanto, en esta metodología, es el instante en el que la muleta entra en contacto con el suelo el que define el inicio de cada ventana, y por tanto, la segmentación. El resultado de la segmentación permite ampliar el número de muestras incorporadas en la base de datos, dado que en vez de trabajar con cada prueba o paciente, se trabajará con cada ciclo como muestra para el proceso de entrenamiento, validación y test de la red neuronal. La Tabla 1 resume el número de muestras total generada.

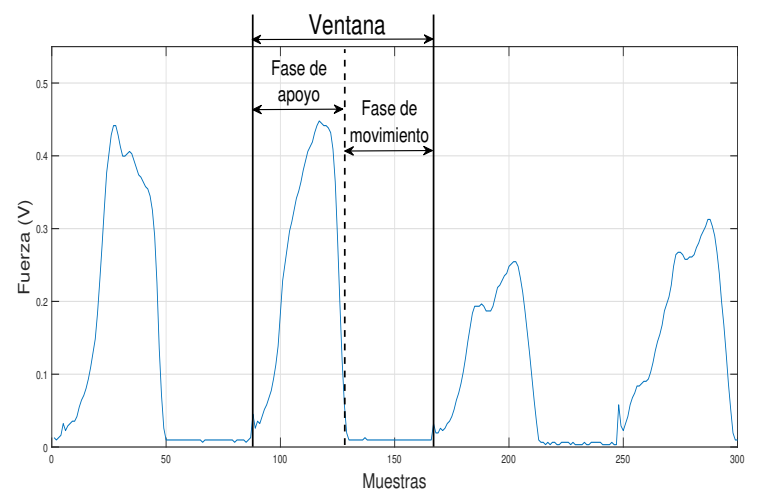

Figura 2: Ciclo de uso de la muleta y sus fases

Las mencionadas muestras son unidades discretas de información, de los cuales se pueden extraer datos estadísticos que caracterizan cada ciclo de la muleta. Para realizar dicha caracterización, que es la última fase del procesamiento, este trabajo se basa en [1], donde se aplican varios operadores a las señales que se obtienen de los sensores integrados en la contera. A diferencia de lo que se hace en el artículo citado, en este caso, se dejan fuera de la caracterización las señales del magnetómetro y los ángulos de Euler (debido a que tratan con ángulos absolutos y pueden derivar a errores), y también el barómetro, ya que su precisión relativa $(0,12 h P a \approx 1 m)$ no es lo suficientemente grande como para detectar variaciones en una prueba de 10 metros en línea recta.

De esta forma, y después de realizar las tres fases de procesamiento de datos, se consiguen 98 características (presentados en la Tabla 2) asociadas a cada ciclo de uso de la muleta. Nótese que estas características están basadas en las propuestas en la literatura. La relevancia relativa de las mis- 
Tabla 2: Datos característicos generados a partir de la información proporcionada por la contera (A=Anteroposterior, $\mathrm{L}=$ Lateromedial).

\begin{tabular}{|c|c|c|c|c|}
\hline Fuente de datos $\rightarrow$ & Acelerómetro & Giróscopo & Ángulos & Sensor \\
\hline Operaciones $\downarrow$ & $(\mathrm{X}, \mathrm{Y}, \mathrm{Z})$ & $(\mathrm{X}, \mathrm{Y}, \mathrm{Z})$ & $(\mathbf{A}, \mathbf{L})$ & de fuerza \\
\hline Media & $\mathbf{X}$ & $\mathbf{X}$ & $\mathbf{X}$ & $\mathbf{X}$ \\
\hline Desviación estándar & $\mathbf{X}$ & $\mathbf{X}$ & $\mathbf{X}$ & $\mathbf{X}$ \\
\hline Varianza & $\mathrm{X}$ & $\mathbf{X}$ & $\mathbf{X}$ & $\mathbf{X}$ \\
\hline Kurtosis & $\mathbf{X}$ & $\mathbf{X}$ & $\mathbf{X}$ & $\mathbf{X}$ \\
\hline Coef. Corr. XY & $\mathbf{X}$ & $\mathbf{X}$ & & \\
\hline Coef. Corr. XZ & $\mathbf{X}$ & $\mathbf{X}$ & & \\
\hline Coef. Corr. YZ & $\mathbf{X}$ & $\mathbf{X}$ & & \\
\hline Coef. Corr. AL & & & $\mathbf{X}$ & \\
\hline Percentil 25 & $\mathbf{X}$ & $\mathbf{X}$ & $\mathrm{X}$ & $\mathbf{X}$ \\
\hline Percentil 50 & $\mathbf{X}$ & $\mathbf{X}$ & $\mathbf{X}$ & $\mathbf{X}$ \\
\hline Percentil 75 & $\mathbf{X}$ & $\mathbf{X}$ & $\mathbf{X}$ & $\mathbf{X}$ \\
\hline Área & $\mathbf{X}$ & $\mathrm{X}$ & $\mathbf{X}$ & $\mathbf{X}$ \\
\hline Rango intercuartil & $\mathbf{X}$ & $\mathbf{X}$ & $\mathbf{X}$ & $\mathbf{X}$ \\
\hline Valor al iniciar el apoyo & & & $\mathbf{X}$ & \\
\hline Valor en Fuerza Máx. & & & $\mathbf{X}$ & \\
\hline Valor al finalizar el apoyo & & & $\mathrm{X}$ & \\
\hline Amplitud & & & $\mathbf{X}$ & \\
\hline Tiempo de ciclo & & & & $\mathbf{X}$ \\
\hline Fase apoyo $\%$ & & & & $\mathbf{X}$ \\
\hline Núm. características & 30 & 30 & 27 & 11 \\
\hline
\end{tabular}

mas ha sido analizada usando la técnica Random Forest.

\section{DISEÑO DEL CLASIFICADOR}

Partiendo de la base de datos definida en la sección 3 , el clasificador tiene como objetivo determinar el valor de la escala SPPB (un número entero entre 0 y 12) que corresponde a cada muestra. A tal fin, el sistema usará las 98 características que extraerá de cada ciclo de la muleta.

Para ello, en este trabajo, se ha optado por utilizar una red neuronal, en concreto, un Perceptrón Multicapa con una única capa oculta. La elección de una red MLP como clasificador se ha realizado en base a los resultados obtenidos en [1]. El artículo citado presenta un procedimiento similar a este, en el que las técnicas Support Vector Machine (SVM) y K Nearest Neighbours (KNN) muestran un comportamiento ligeramente inferior a las redes neuronales artificiales (ANN).

Dicha red está compuesta por 98 neuronas de entrada (debido a la caracterización realizada en la sección 3.2), 13 neuronas de salida (uno por cada valor en la escala SPPB) y 10 neuronas en la capa oculta con la función de activación tangente hiperbólica-sigmoidal (Figura 3). La definición del número de neuronas en la capa oculta se ha basado en pruebas experimentales que han mostrado resultados óptimos con un número de 10 neuronas en dicha capa.

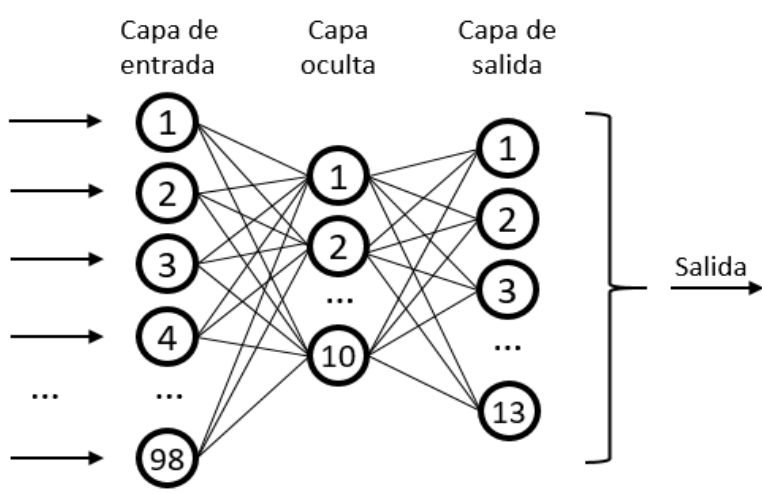

Figura 3: Estructura de la red neuronal

Una vez definida la estructura de la red, a continuación, se han preparado los datos con el objetivo de realizar un adecuado proceso de entrenamiento y validación. Para ello, la base de datos se ha dividido en dos conjuntos: uno para entrenamiento, y otro para test.

Para realizar dicha división, se ha tenido en cuenta el número de pruebas que se tienen por cada etiqueta. De esta forma, se han incluido en el conjunto de entrenamiento las muestras correspondientes a un $80 \%$ de las pruebas por cada etiqueta, y el resto de las ventanas, se han utilizado para el test. Cabe mencionar, que en este caso, tanto el número de pruebas por cada etiqueta, como el número de muestras por cada 
Tabla 3: Matriz de confusión del conjunto de datos para test.

\begin{tabular}{|c|c|c|c|c|c|c|c|c|c|c|c|c|c|}
\hline $\begin{array}{c}\text { Valores predichas } \rightarrow \\
\text { Valores reales } \downarrow\end{array}$ & 0 & 1 & 2 & 3 & 4 & 5 & 6 & 7 & 8 & 9 & 10 & 11 & 12 \\
\hline 0 & 0 & 0 & 0 & 0 & 0 & 0 & 0 & 0 & $\begin{array}{l}0 \\
\end{array}$ & 0 & 0 & 0 & 0 \\
\hline 1 & 0 & 0 & 0 & 0 & 0 & 0 & 0 & 0 & 0 & 0 & 0 & 0 & 0 \\
\hline 2 & 0 & 0 & 0 & 0 & 0 & 0 & 0 & 0 & 0 & 0 & 0 & 0 & 0 \\
\hline 3 & 0 & 0 & 0 & 12 & 0 & 0 & 2 & 1 & 0 & 0 & 0 & 0 & 0 \\
\hline 4 & 0 & 0 & 0 & 0 & 0 & 0 & 12 & 0 & 5 & 9 & 0 & 0 & 0 \\
\hline 5 & 0 & 0 & 0 & 1 & 0 & 7 & 9 & 2 & 0 & 0 & 0 & 0 & 0 \\
\hline 6 & 0 & 0 & 0 & 0 & 0 & 16 & 15 & 0 & 2 & 2 & 0 & 5 & 0 \\
\hline 7 & 0 & 0 & 0 & 5 & 3 & 0 & 5 & 9 & 2 & 0 & 1 & 0 & 0 \\
\hline 8 & 0 & 1 & 0 & 2 & 0 & 0 & 6 & 0 & 1 & 7 & 8 & 0 & 0 \\
\hline 9 & 0 & 0 & 0 & 0 & 1 & 1 & 6 & 0 & 9 & 17 & 0 & 0 & 0 \\
\hline 10 & 0 & 0 & 0 & 0 & 0 & 0 & 5 & 0 & 0 & 0 & 4 & 1 & 0 \\
\hline 11 & 0 & 0 & 0 & 0 & 2 & 0 & 2 & 0 & 0 & 0 & 2 & 5 & 0 \\
\hline 12 & 0 & 0 & 0 & 0 & 0 & 0 & 0 & 0 & 0 & 0 & 0 & 0 & 0 \\
\hline
\end{tabular}

prueba es variable y que por tanto, no ha sido posible conseguir conjuntos equilibrados. La Tabla 1 presenta el número de muestras que constituyen cada conjunto.

Finalmente, una vez que se han preparado la estructura de la red y los datos, se ha empleado el algoritmo de Regularización Bayesiana para entrenar la red. Dicho entrenamiento se ha realizado 10 veces para tener en cuenta el efecto de la inicialización aleatoria de las neuronas y finalmente, se ha seleccionado la red que mejores resultados ha proporcionado.

\section{RESULTADOS}

En esta sección se detallarán los resultados más relevantes obtenidos por la red. A tal fin, se han considerado dos escenarios de validación: uno con los mismos datos que se han empleado en el entrenamiento y otro, con los datos que se han dejado para test.

Con el primer conjunto, el que incluye los datos empleados en el entrenamiento, la red ha obtenido un porcentaje de aciertos del 97,9\%. Este resultado indica que el entrenamiento de la red ha sido el adecuado, y ésta ha logrado adaptarse al conjunto de muestras de entrenamiento.

Sin embargo, con el conjunto de test, el porcentaje de aciertos ha sido de $34,1 \%$. Estos resultados indican un porcentaje muy bajo de aciertos, que reflejan que la red entrenada no es capaz de generalizar a partir de los datos de entrenamiento. Con el fin de estudiar este fenómeno, se han analizado los ratios de acierto para cada escala SPPB. La Tabla 3 muestra la matriz de confusión que se ha obtenido con este segundo conjunto de datos.

Los resultados muestran que, en el caso del con- junto de test, la red categoriza adecuadamente algunas muestras (por ejemplo, las asociadas a la SPPB de valor 3 o un porcentaje elevado de la 9). Sin embargo, en muchas ocasiones, la red confunde la escala de un paciente por la inmediatamente anterior o la siguiente, como sucede en el caso de la SPPB de valor 5 o 6 . En general, se aprecia que la matriz de confusión tiene una diagonal predominante, pero que en la zona central de la tabla existe una dispersión importante, principalmente entre las categorías adyacentes.

Es importante detallar que estos resultados no están causados por el proceso de entrenamiento. La configuración mostrada es la que mejor resultado ofrece, tras realizar ensayos con diferentes algoritmos de entrenamiento y conjuntos de validación. En este sentido, y tras analizar el conjunto de datos capturado, una de las problemáticas que se ha detectado es la gran variabilidad existente entre los valores característicos extraídos de pacientes con una SPPB similar. La Figura 4 muestra como ejemplo la distribución que tienen la media del acelerómetro y la media del giróscopo en el eje $x$, según el valor SPPB correspondiente a cada muestra. En ella se observa que dentro de cada etiqueta la variabilidad de los valores característicos es significativa, lo cual hace que el rango en el que se encuentren los datos no pueda asociarse directamente a un valor en la escala SPPB. Este comportamiento se repite en la mayoría de los datos característicos extraídos, lo cual indica que dentro de cada etiqueta existe una variabilidad notable entre pacientes.

Este fenómeno es habitual en EM, dado que la enfermedad puede evolucionar de formas muy diversas. Esto genera una mayor complejidad en el análisis de datos, y es la razón por la que en la Matriz de Confusión ilustrada en la Tabla 3 la red interpreta las señales de entrada en ocasiones con 

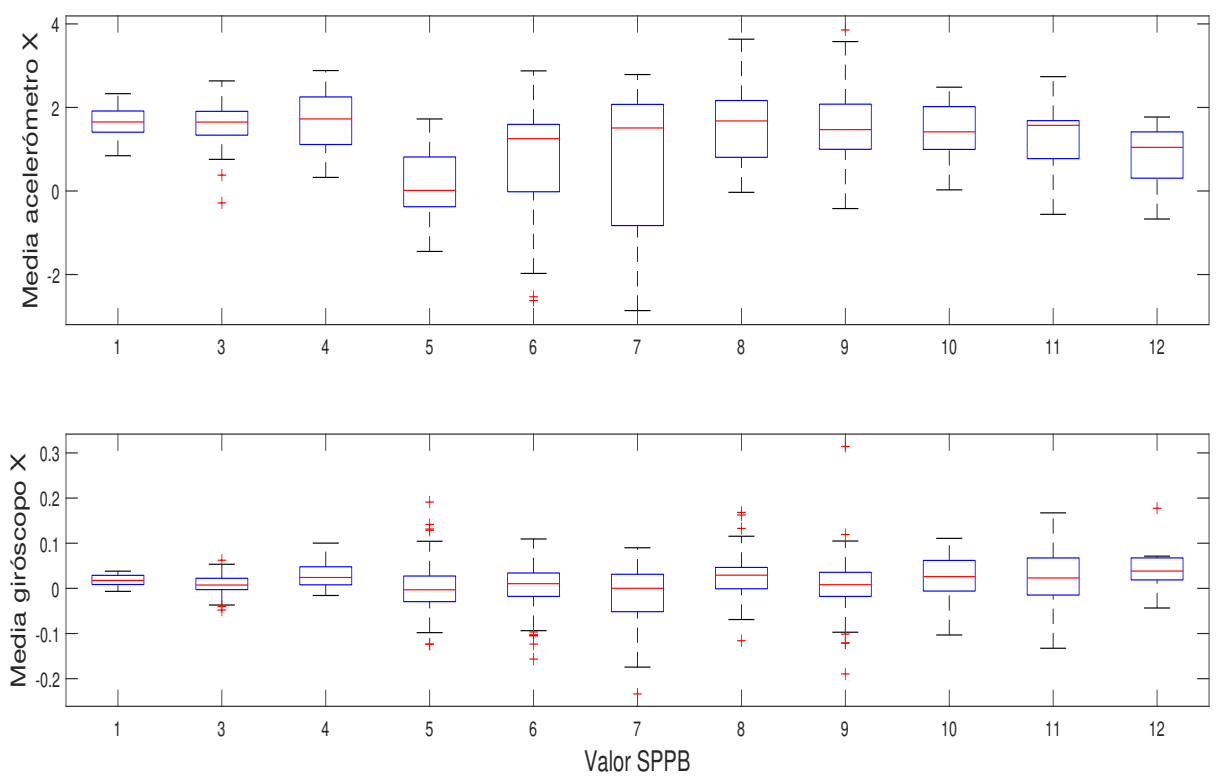

Figura 4: Distribución de la media del acelerómetro y de la media del giróscopo en el eje $x$ según el valor SPPB de cada muestra

valores de SPPB cercanos al valor real.

Es por ello que, si bien el uso de técnicas inteligentes se considera una técnica de potencial para esta enfermedad, es necesario abordar esta problemática desde otro enfoque: incorporando un mayor número de datos que permita caracterizar un mayor conjunto de pacientes; o apostando por un enfoque personalizado, en el que la caracterización del estado del paciente se base en su evolución personalizada.

\section{CONCLUSIONES}

Una adecuada valoración del estado funcional de pacientes con Esclerosis Múltiple ha demostrado ser esencial para personalizar las terapias y mejorar así la calidad de vida de dichos pacientes [6].

Tradicionalmente, esta valoración se ha realizado utilizando escalas clínicas normalizadas, como la SPPB. Sin embargo, el obtener esos valores es una tarea laboriosa, por lo que la periodicidad entre las sesiones de evaluación es elevada [5].

Con el fin de agilizar dicho proceso, en este trabajo, se ha diseñado un clasificador que partiendo de los datos que proporciona una contera inteligente acoplada al dispositivo de ayuda técnica del paciente, pretende determinar el valor de la escala SPPB que corresponde a dicho paciente.

Para ello, en primer lugar, se ha generado una base de datos, partiendo de pruebas de 10 metros realizadas con pacientes de EM. A continuación, se ha definido la estructura de una red neuronal, se han preparado los conjuntos de datos de entrenamiento y test, y se ha entrenado la red diseñada. Finalmente, se ha validado dicha red obteniendo un porcentaje de aciertos de 97,9\% con las mismas muestras empleadas en el entrenamiento, y un porcentaje de aciertos de $34,1 \%$ con los datos que se han dejado para test.

Estos resultados muestran que la red no es capaz de generalizar, ya que no ha identificado correctamente aquellas muestras que desconocía. Esto puede deberse por un lado al desequilibrio de la base de datos de la que se dispone; sin embargo, teniendo en cuenta la problemática que se está tratando, es probable que un enfoque general no llegue a dar resultados satisfactorios, y sea necesario abordar el problema desde un enfoque individual.

\section{Agradecimientos}

Este trabajo ha sido financiado por la Universidad del País Vasco UPV/EHU (GIU19/045), FEDER/Ministerio de Ciencia, Innovación y Universidades - Agencia Estatal de Investigación/ DPDPI2017-82694-R, y la ayuda FPU del Ministerio de Ciencia, Innovación y Universidades (FPU19/04874).

Los autores agradecen también a la Asociación de Esclerosis Múltiple de Bizkaia (ADEMBI) por su colaboración.

\section{English summary}




\section{APPLICATION OF NEURAL NET- WORKS FOR THE ASSESSMENT OF PEOPLE WITH MULTIPLE SCLEROSIS}

\begin{abstract}
Multiple Sclerosis is a neurological degenerative disease with no cure. However, personalized rehabilitation therapies have demonstrated to be effective to reduce the effect of symptoms and improving this way the life quality of patients. To design such therapies, it is essential to know the functional status of each patient, which is usually a laborious task. For that reason, in this work a status classifier for Multiple Sclerosis patients is developed. Artificial neural networks are used for this task, which work with a database obtained by a Smart Tip for developing a system to determine the functional status of patients.
\end{abstract}

Keywords: Multiple Sclerosis, Smart Tip, Patients assessment, Functional status classifier, Machine Learning, Artificial Neural Networks.

\section{Referencias}

[1] Brull, A., Lucas, S., Zubizarreta, A., Cabanes, I., Portillo, E., Rodriguez-Larrad, A., "A Machine Learning Approach to Perform Physical Activity Classification Using a Sensorized Crutch Tip", IEEE Access, vol 8, pp 210023-210034, 2020.

[2] Brull, A., Zubizarreta, A., Cabanes, I., Rodriguez-Larrad, A., "Sensorized Tip for Monitoring People with Multiple Sclerosis that Require Assistive Devices for Walking", Sensors, vol 20(15), pp 4329, 2020.

[3] Chamorro-Moriana, G., Sevillano, J., RidaoFernández, C., "A Compact Forearm Crutch Based on Force Sensors for Aided Gait: Reliability and Validity", Sensors, vol 16(6), pp 925, 2016.

[4] Creagh, A.P., et al., "Smartphone- and Smartwatch-Based Remote Characterisation of Ambulation in Multiple Sclerosis during the Two-Minute Walk Test", IEEE Journal of Biomedical and Health Informatics, vol 25(3), pp 838-849, 2020.

[5] Flachenecker, P., "Clinical implications of neuroplasticity - the role of rehabilitation in multiple sclerosis", Frontiers in Neurology, vol 6(36), 2015.

[6] Latimer-Cheung, A.E., et al., "Effects of Exercise Training on Fitness, Mobility Fatigue, and Health-Related Quality of Life Among Adults With Multiple Sclerosis: A Systematic Review to Inform Guideline Development", Archives of Physical Medicine and Rehabilitation, vol 94(9), pp 1800-1828, 2013.

[7] McGinnis, R.S., et al., "A machine learning approach for gait speed estimation using skin-mounted wearable sensors: From healthy controls to individuals with multiple sclerosis", PLOS ONE, vol 12(6), 2017.

[8] Prakash, C., et al, "Recent developments in human gait research: parameters, approaches, applications, machine learning techniques, datasets and challenges", Artificial Intelligence Review, vol 49, pp 1-40, 2018.

[9] Sardini, E., Serpelloni, M., Lancini, M., "Wireless Instrumented Crutches for Force and Movement Measurements for Gait Monitoring", IEEE Transactions on Instrumentation and Measurement, vol 64(12), pp 3369-3379, 2015.

[10] Sociedad Española de Neurología (SEN). Informe del Día Nacional de la EM.

[11] Souza, A., Kelleher, A., Cooper, R., Cooper, R.A., Iezzoni, L.I., Collins, D.M., "Multiple sclerosis and mobility-related assistive technology: Systematic review of literature", The Journal of Rehabilitation Research and Development, vol 47(3), pp 213-223, 2010.

[12] Spain, R., et al., "Body-worn motion sensors detect balance and gait deficits in people with multiple sclerosis who have normal walking speed", Gait Posture, vol 35(4), pp 573-578, 2012.

[13] Sun, R., et al., "Assessment of Postural Sway in Individuals with Multiple Sclerosis Using a Novel Wearable Inertial Sensor", Digital Biomarkers, vol 2, pp 1-10, 2018.

[14] Zhao, Y., et al., "Exploration of machine learning techniques in predicting multiple sclerosis disease course", PLOS ONE, vol $12(4), 2017$. 
(c) (1) () $\stackrel{\text { Submitted }}{2021}$ by the $\begin{aligned} & \text { authors. } \\ & \text { fossible }\end{aligned}$ ${ }_{\text {BY NC SA }}$ open access publication under the terms and conditions of the Creative Commons Attribution CC BY-NC-SA 4.0 license (https://creativecommons.org/licenses/by-ncsa/4.0/deed.es). 